\title{
Multicast in UMTS: Adopting TCP-Friendliness
}

\author{
Antonios Alexiou ${ }^{1,2}$, Christos Bouras ${ }^{1,2}$, Andreas Papazois ${ }^{2}$ \\ 'Research Academic Computer Technology Institute, N. Kazantzaki str., \\ 26500 Patras $_{s}$ Greece \\ ${ }^{2}$ Computer Engineering and Informatics Department, \\ University of Patras, 26500 Patras, Greece \\ alexiua@cti.gr, bouras@cti.gr, papazois@ceid.upatras.gr
}

\begin{abstract}
In this paper, we present a novel mechanism for the multicast congestion control over UMTS networks. The proposed mechanism is based on the well known TCP-Friendly Multicast Congestion Control (TFMCC) scheme. The key challenge in the design of the new scheme lies in improving the TFMCC mechanism to cope with the packet losses caused by either the temporary or the permanent degradation of the wireless channels. The proposed scheme introduces minor modifications in the UMTS nodes with respect to the computing power of the mobile terminals. Finally, our approach is implemented in the ns- 2 network simulator and is evaluated it under various conditions.
\end{abstract}

Keywords: multicast, congestion control, TFMCC, TCP-friendliness, UMTS.

\section{Introduction}

Universal Mobile Telecommunications System (UMTS) constitutes the most prevalent standard of the $3 \mathrm{G}$ cellular networks. UMTS networks promise the provision of advanced services along with high data rates. Despite the high capacity that UMTS networks provide, the expected demand will certainly overcome the available resources. This is the reason why multicast transmission is one of the major goals for UMTS and 3G networks in general [1].

Multicast is an efficient method for data transmission to multiple destinations. Its advantage is that sender's data are transmitted only once over links which are shared along the paths to a targeted set of destinations. On the other hand, congestion control is a policy that regulates the source transmission rate according to the network congestion. The IP multicast, contrary to the Transmission Control Protocol (TCP), does not implement any congestion control. This means that the coexistence of multicast traffic and TCP traffic may lead to unfair use of network resources. In order to prevent this situation, the deployment of multicast congestion control is indispensable. This kind of congestion control is well-known as TCP-friendliness [2].

The adoption of a multicast congestion control in cellular networks poses an additional set of challenges which are related to the existence of wireless links and mobile terminals. In the first place, all the algorithms for congestion control treat the 
packet loss as a manifestation of network congestion. This assumption may not apply to networks with wireless links, in which packet loss is often induced by noise, wireless link error or reasons other than congestion [3]. Another requirement is that the mobile terminals' computing power cannot afford complicated functions. Consequently, such operations should be avoided to be held on mobile equipment.

In this paper, we present a novel mechanism for the multicast congestion control over UMTS networks. The proposed mechanism is based on the well known TFMCC scheme. TFMCC is an equation-based multicast congestion control mechanism simple enough so as to meet a prime objective for UMTS multicast services, that is scalability to applications with thousands of receivers. In our proposed mechanism, the TFMCC scheme is partly modified and extended in order to support the particularities of the UMTS Terrestrial Radio-Access Network (UTRAN). The major problem of the applicability of TFMCC over UMTS is the Current Limiting Receiver (CLR) problem. Minor modifications in the UMTS architecture are introduced by our proposed scheme.

This paper is structured as follows: Section 2 provides an overview of the work related to the scientific domain. In Section 3, we briefly present the TFMCC algorithm and describe the problem of the applicability of congestion control over the wireless access networks. Section 4 is dedicated to the proposed congestion control mechanism. Section 5 describes the simulation experiments. Finally, some concluding remarks and planned next steps are stated in Section 6 .

\section{Related Work}

Multicast congestion control problem in fixed networks is still a domain of active research and a lot of solutions have been proposed until now. We use two distinct properties to classify the existing approaches [4], [5]:

- The rates delivered to the receivers in a session. The existing approaches fall into three categories: single-rate, multi-rate and layered.

- The place where adaptation is performed. It is either at the end systems (end-to-end service), or at the intermediate network nodes (active service).

A technical problem of major importance in multicast congestion control is scalability. When the source receives a negative feedback of congestion notification inside the network, it regulates its transmission rate. In order to avoid a feedback implosion, the majority of the researchers, like the authors of [6], suggest that the receiver of the worst congestion level should be selected as the representative. In this approach, only the representative transmits feedback information for congestion control and the number of feedbacks is limited.

In contrast to the multicast congestion control problem in fixed networks, no specific solutions and algorithms have been proposed for the variation of this problem in cellular networks. Despite radio network congestion being a widely recognized and identified problem, few relevant studies have been published. The most strongly related publication is [7]. However, this publication refers to the extended class of 
wireless access networks (including WLANs) and it is not well aligned with 3GPP specifications for the UMTS cellular networks.

\section{Overview of Basic Concepts}

\subsection{TFMCC Mechanism}

TFMCC is a well known equation-based multicast congestion control mechanism which is fair towards competing TCP flows, i.e. is TCP-friendly. TFMCC belongs to the class of single rate congestion control schemes and applies at the end systems (end-to-end service). Such schemes inevitably do not offer multiple transmission rates as layered schemes do. However, they are much simpler so as to offer scalability to applications with thousands of receivers.

TFMCC uses a control equation derived from a model of TCP's long term throughput to directly control the sender's transmission rate. The loss event rate and the Round-Trip Time (RTT) are the parameters that define this target throughput. Each receiver calculates its target throughput and considers it as the acceptable sending rate from the sender to itself.

TFMCC uses a feedback scheme which allows the receiver calculating the slowest transmission rate to always reach the sender. This scheme is based on the concept of the Current Limiting Receiver (CLR). The CLR is the receiver that the sender believes currently has the lowest expected throughput of the multicast group. Moreover, the TFMCC design ensures that the sender gets feedback from the receivers experiencing the worst network conditions without being overwhelmed by feedback (feedback implosion is suppressed).

For full details of TFMCC, we refer the reader to [2].

\subsection{MBMS Service in UMTS}

In the beginning of the current decade, the UMTS standardization body, 3G Partnership Project (3GPP), started the standardization of Multimedia Broadcast/Multicast Service (MBMS) framework. As the term Broadcast/Multicast implies, two types of service mode exist in MBMS service: the broadcast and the multicast. In the broadcast mode, data are delivered to all the receivers roaming in a specific area. On the other hand, in the multicast mode the receivers have to declare their interest for the data reception. The service then decides whether the user may receive data or not. During the rest of our analysis we will focus on the multicast mode. The multicast mode is the most complicated and also covers all the aspects of the broadcast mode.

From the physical point of view, the UMTS network architecture is organized in two domains: the User Equipment UE and the Public Land Mobile Network (PLMN). The UE is used by the subscribers to access the UMTS services while the PLMN is a network established by an operator to provide mobile telecommunications services to 
the public. The PLMN is further divided into two land-based infrastructures: the UMTS Terrestrial Radio-Access Network (UTRAN) and the Core Network (CN). The UTRAN handles all radio-related functionalities. The $\mathrm{CN}$ is responsible for maintaining subscriber data and for switching voice and data connections.

The UTRAN consists of two kinds of nodes: the first is the Radio Network Controller (RNC) and the second is the Node B. The Node B is connected to the UE via the $U u$ interface and to the $R N C$ via the Iub interface. The $U u$ is a radio interface based on the Wideband Code Division Multiple Access (WCDMA) technology. A single RNC with all the Node Bs connected to it, is called Radio Network Subsystem (RNS).

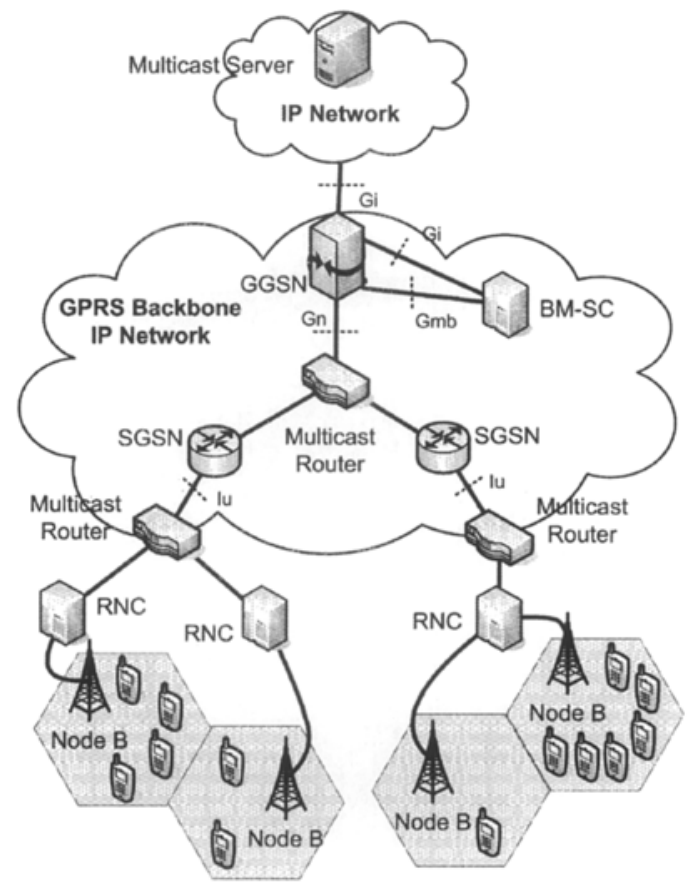

Fig. 1. MBMS architecture for UMTS using IP multicast.

The CN is logically divided into the Circuit-Switched (CS) domain and the PacketSwitched (PS) domain. The PS-domain is more relevant and therefore, in the remainder of this paper, more attention will be devoted to the PS-functionality. The PS-domain of the CN consists of two kinds of General Packet Radio Service (GPRS) Support Nodes (GSNs), namely Gateway GSN (GGSN) and Serving GSN (SGSN). The SGSN is the centerpiece of the PS-domain. It provides routing functionality, it manages a group of RNSs and it interacts with the Home Location Register (HLR) which is a database permanently storing subscribers' data. The SGSN is connected to GGSN via the Gn interface and to RNCs via the Iu interface. GGSN provides the 
interconnection between the UMTS network and the external Packet Data Networks (PDNs) like the Internet [1].

Fig. 1 illustrates the basic MBMS architecture for UMTS. The most significant modification of the UMTS architecture is the addition of a new node called Broadcast Multicast--Service Center (BM-SC). The BM-SC is a data source unique to MBMS. In this node the MBMS data are scheduled and interfaces are provided for the interaction with the content provider. The BM-SC may authorize and charge the content provider. At this point, it must be clarified that the data source may not originate from an external PDN, but may also originate from within the UMTS network.

In order to reduce the implementation costs, the MBMS has been designed to introduce only minor changes to existing radio and core network architectures. For simplicity reasons, in our analysis, we will consider the functionality of the BM-SC incorporated in the GGSN.

\subsection{CLR Selection Problem}

The traditional congestion control mechanisms treat the packet loss as manifestation of buffer overflow in the network nodes, i.e. as network congestion. Consequently, the action taken in order to resolve this situation is the reduction of the sender's transmission rate. This assumption may not apply to networks with wireless links, in which packet loss is often induced by noise, wireless link error or reasons other than network congestion. As a consequence, the network reaction should not be a drastic reduction of the sender's transmission rate [3]. The wireless-caused packet loss will eventually be resolved after the end of the fading period, without a transmission rate regulation.

Obviously, the wireless channel degradation may affect the performance of the TFMCC mechanism. If a lot of UEs participate in the multicast group, there is a high probability that at a given time, a UE suffers from fading. Eventually, the wirelesschannel degradation will cause a significant and steady degradation of the performance of the TFMCC mechanism and of the multicast service. During this analysis we shall refer to this problem as the CLR selection problem.

\section{The Proposed Mechanism}

As we have already mentioned, the proposed mechanism follows a design very similar to that of the TFMCC scheme. Nevertheless, new functionality has been added to the existing mechanism in order to deal with the CLR selection problem.

The basic principles that govern the proposed mechanism are the following:

1. Each UE measures its packet loss rate using the packet loss history scheme of TFMCC.

2. Each Node B measures its packet loss rate. This information is written to the heading of the data packets and is then read by the UEs. This is a new functionality which combats the CLR selection problem in UMTS networks. This functionality does not exist in the TFMCC scheme and is explained below. 
3. Each UE measures or estimates the RTT to the multicast server. This is achieved through an approach inherited from TFMCC. In more detail, timestamped feedback is sent to the multicast server. The server then echoes the timestamp and the corresponding UE_id in the header of a data packet. This approach causes minor traffic overhead in the network.

4. Each UE uses a control equation to calculate an acceptable sending rate from the sender back to it. The input parameters for the control equation are the loss rate and the RTT measured by the UE.

5. The feedback scheme of TFMCC is adopted. This scheme has devised a way in that the feedback from the receiver calculating the slowest transmission rate always reaches the sender. In addition, the feedback is filtered using randomized timers in order to avoid a feedback implosion.

In the proposed mechanism, the nodes located at the border between wireless and wired network (i.e. the Node Bs) have an additional responsibility. This responsibility is to provide the receivers (i.e. the UEs) with information about their measured packet loss. This means that each UE is informed by its serving Node $\mathrm{B}$, of the packet loss that the Node B measures. This information is piggybacked in the data packets of a multicast session.

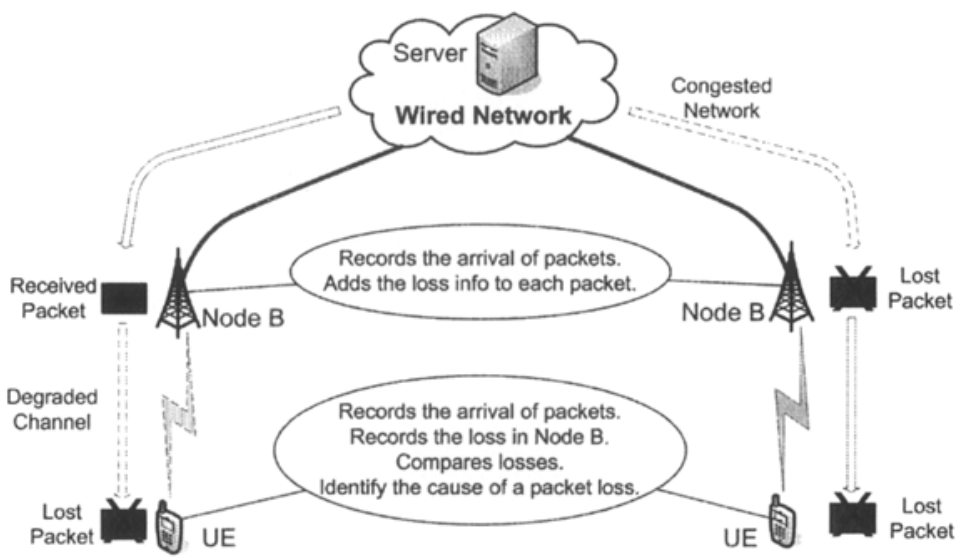

(a)

(b)

Fig. 2. Packet losses at the UE: (a) Packet loss due to wireless channel degradation.

(b) Packet loss due to network congestion.

This additional functionality of the Node Bs, permits each UE to identify the reason of a packet loss. The UE compares the packet loss received from Node B with its measured packet loss. In general, the following cases are distinguished:

- When the two values differ, the UE can conclude that the reason for the difference is losses at the wireless link caused by wireless channel degradation. This kind of packet loss is not related to the network congestion and, consequently, the reduction of the transmission rate will not affect this packet loss. In this case, the 
packet loss is not accounted at the CLR selection. Fig. 2(a) visualizes this functionality of the UE.

- On the other hand, when both the Node B and the UE encounter a packet loss, this packet loss is considered to be caused due to network congestion. Consequently, this kind of packet loss is taken into consideration during the CLR selection. This scenario is depicted in Fig. 2(b).

When a permanent degradation occurs on the wireless link, the buffer of the Node B will overflow and some packets will be rejected. Normally, this UE should be a CLR-candidate. In the proposed mechanism, during permanent channel degradation, the Node B counts the rejected packets as general packet losses which happened due to network congestion. These packets are taken into account by the UE during the CLR selection. This mechanism assures that this permanent degradation is not hidden from the UE. In fact, the UE is informed of the packet losses caused by buffer overflow. This functionality makes our proposed mechanism suitable not only with the CLR selection problem, but also with the permanent degradation of the wireless channel.

\section{Experiments}

\subsection{Simulation Environment}

For the verification of the proposed mechanism the ns-2 network simulator [8] along with its EURANE extension were used. The Enhanced UMTS Radio Access Network Extensions (EURANE) for ns-2 [9] comprises of extensions for the support of UMTS network functionality. Given that the ns-2 simulator does not support the multicast transmission in UMTS, we implemented the multicast packet forwarding mechanism described [10]. The next step was the installation of the TFMCC scheme. The codes used to implement and evaluate the TFMCC by the authors of [2].

Finally, the generic TFMCC was modified and extended in order to support the UMTS environment. In more detail, the implementation of the TFMCC was enhanced in order to support the functionality of the Node B and the UE as described in Section 4. The Node B implementation was modified in order to provide the UEs with information about its measured packet loss. This means that each UE is informed by its serving Node B, of the packet loss that the Node B measures. This information is piggybacked in the data packets of a multicast session. One bit in the header of the data packet is enough for the provision of this information. On the other hand, the UE implementation was modified in order to read this bit and to take the decision whether a packet loss should be accounted at the calculation of its acceptable sending rate.

\subsection{TCP-Friendliness}

The first aspect that we examined was the TCP-friendliness of TFMCC. In more detail, we considered the fairness of TFMCC towards the competing TCP flows when 
they share wired or wireless links. Below, we present the TFMCC behavior in a noncongested and in a congested UMTS network.

In the first place, fairness was analyzed using a non-congested UMTS network. We monitored the throughput over a wireless link connected a UE with its serving Node B. We supposed that UE belongs to a multicast group and receives TFMCC traffic. At the same time, this UE receives TCP traffic from an external node. Fig. 3 illustrates the throughput of TFMCC flow against that of the TCP flow.

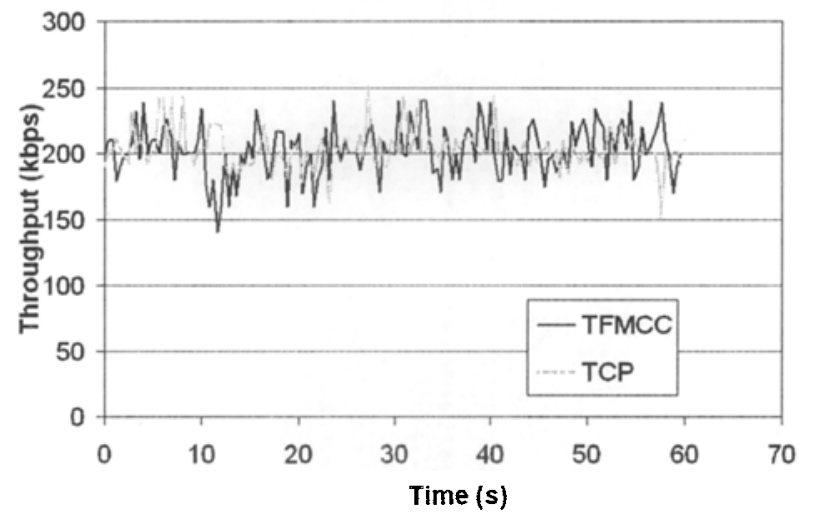

Fig. 3. TFMCC flow vs. TCP flow in a non-congested UMTS network.

Due to our initial assumption that no congestion exists, the capacity of the wireless link poses a threshold of $384 \mathrm{kbps}$ for the throughput of the flows towards the examined UE. As it was expected, the available bandwidth is fairly shared between the flows. Fig. 3 confirms that the average throughput of TFMCC flow closely matches the average TCP throughput.

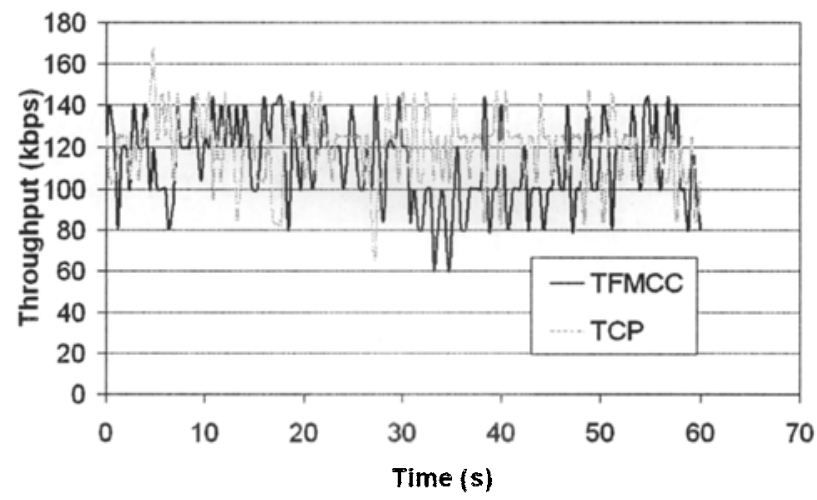

Fig. 4. TFMCC flow vs. TCP flow in a single-bottleneck UMTS network. 
The next step of our experiment was to examine the fairness of the mechanism in a congested UMTS network. We considered a single-bottleneck topology and the bottleneck was applied over a link which connects an SGSN with an RNC node (Iu interface).

Fig. 4 shows the throughput of a TFMCC flow against two sample TCP flows (out of 15). The average throughput of TFMCC closely matches the average TCP throughput. Moreover, TFMCC achieves a smoother rate than the TCP.

Similar results can be obtained for many other scenarios. For example, if we suppose that congestion exists over a wired UMTS interface.

\subsection{Responsiveness to Changes}

An important concern in the design of congestion control protocols is their responsiveness to changes in the network conditions. This behavior was investigated using the single bottleneck topology of used in the previous experiment.

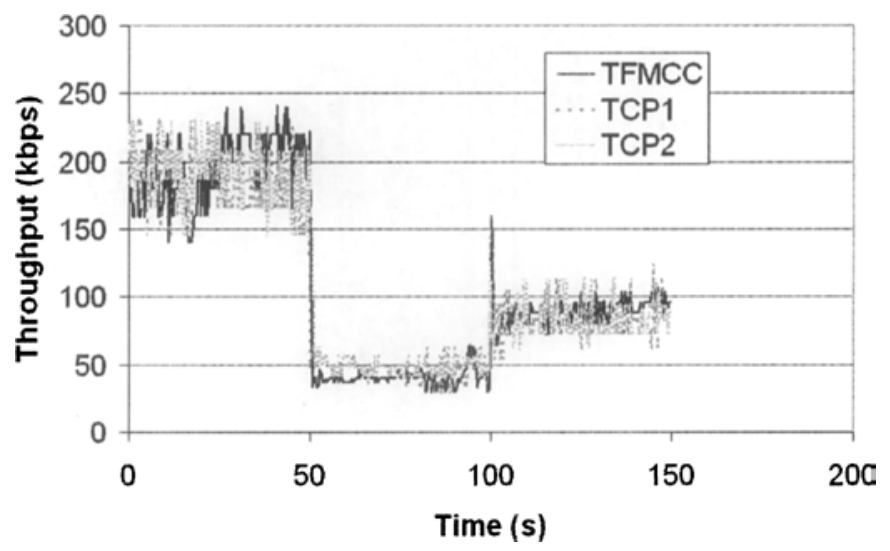

Fig. 5. Responsiveness to changes in the loss rate.

During the simulation we changed the applied loss rate of the bottleneck link. The simulation lasted 150 seconds. During this time interval three different loss rates were applied on the Iu interface. The TFMCC flow was monitored along with two TCP flows sharing the bottleneck link. The results of the simulation for the three competing flows are presented in Fig. 5.

As shown in Fig. 5, TFMCC matches closely the TCP throughput at all three loss levels. Moreover, the adaptation of the sending rate is fast enough. Actually, the simulator logs show that the UEs need $1500-2000 \mathrm{~ms}$ after the change of the loss rate in order to adapt to the new loss rate. These figures of response time are close enough to the corresponding time of TCP (about 1000-1500 ms).

A similar simulation setting was used in order to investigate the responsiveness to changes in the RTT. The results are similar to those above. The above experiment 
confirms the excellent reactivity of the TFMCC to changes in congestion level of the UMTS network. Moreover, it confirms that during the application of TFMCC over the UMTS the properties and the benefits of this scheme are not affected.

\subsection{Reaction to Wireless Channel Degradation}

The next concern of our experiments was the evaluation of the proposed scheme when wireless-caused packet losses occur. We simulated a UMTS network and assumed a degradation of the wireless channels. In more detail, we simulated the wireless channel degradation by applying an error rate over the packets transmitted via the wireless links. We examined the proposed scheme for different number of UEs belonging in the multicast group.

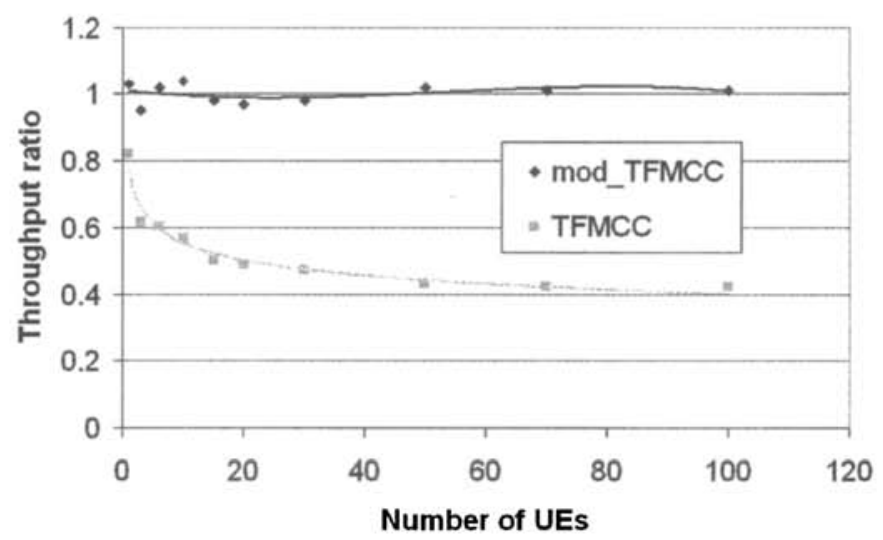

Fig. 6. Throughput of our proposed scheme vs. TFMCC when wireless packet losses occur.

In Fig. 6, our proposed scheme is referred as modified TFMCC (mod_TFMCC). On the other hand, the typical TFMCC algorithm is referred as TFMCC. The horizontal axis shows the number of the UEs belonging in the examined multicast group. Both mechanisms were examined for up to 100 UEs participating in the multicast group. The vertical axis shows the average throughput which is normalized to the corresponding TCP one. The results when $5 \%$ wireless-caused packet loss is applied are presented in Fig. 6.

The results depicted in Fig. 6 confirm the excellent behavior of our proposed scheme when wireless channel degradation occurs. The wireless-caused packet losses can be identified correctly at the UEs and be ignored at the calculation of the acceptable sending rate. This means that the CLR selection problem can be overcome and significant improvement is added on the TFMCC application over the UMTS. 


\subsection{Permanent Wireless Channel Degradation}

The last concern of our experiments was the evaluation of the proposed scheme when wireless-caused packet losses occur in a permanent manner. We examined the behavior of the modified TFMCC when a wireless channel is permanently degraded so as to lead to buffer overflow and packet rejections in the corresponding Node B. In the simulated UMTS network of this setting we assumed a permanent degradation of the wireless link that connects a specific UE with the corresponding Node B.

In more detail, we simulated the wireless channel degradation by applying an error rate of $50 \%$ over the packets transmitted via the corrupted wireless link. In the beginning of the simulation, no wireless channel degradation occurred. After 50 seconds of simulation, we applied the error rate over the wireless channel connecting the examined UE. We monitored the changes over the throughput of the corrupted wireless link for 100 seconds. The results of our experiment are presented in Fig. 7.

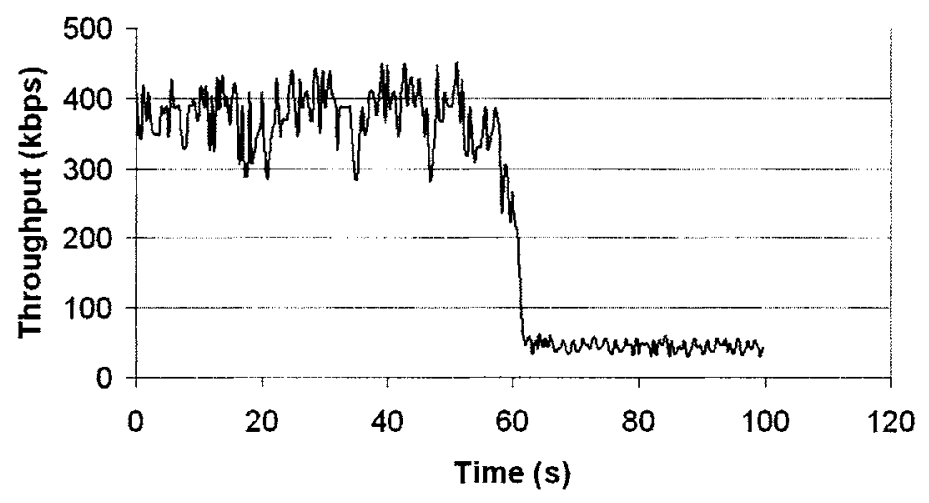

Fig. 7. Throughput of our proposed scheme vs. TFMCC when wireless packet losses occur.

The simulation results prove that our proposed scheme reacts to the permanent wireless-channel degradation. The packet losses in the Node B are considered as network congestion and are not ignored during the calculation of the acceptable sending rate in the UE. Eventually, this kind of packet losses causes reduction of the transmission rate. It took about 10 seconds to adapt to the new network conditions, but this time interval may differ according to the bit-rate of the transmission and the size of the buffer in Node B.

\section{Conclusions and Future Work}

We have described a congestion control scheme for the multicast transmission over UM'TS. The proposed scheme was evaluated through simulation experiments and concluded that it preserves the benefits of TFMCC algorithm over the UMTS cellular 
network. The mechanism is fair towards competing TCP flows and has very good reactivity to changes in the congestion level.

Additionally, simulation experiments were performed in order to examine the proposed scheme against the CLR selection problem. The experiments proved that the wireless-caused packet losses can be identified correctly at the UEs, whereas the permanent wireless channel degradation is considered as network congestion.

Last but not least, the proposed scheme introduces minor modification in the UMTS architecture and respects the limited computing power of the UEs and no demanding operation is introduced in those network nodes.

The step that follows this work is the evaluation of different congestion control schemes for UMTS networks. Other multicast congestion control schemes will be investigated and modified in order to meet the UMTS requirements. Furthermore, we will try to formulate a multicast group control mechanism dedicated for the UMTS networks. In some cases, permanent wireless channel degradation may cause a large reduction to the transmission rate and eventually multicast service degradation. It will be specified under which circumstances wireless channel degradation will cause rejection of a corrupted UE from a multicast group.

\section{References}

1. Holma, H. and Toskala, A. WCDMA for UMTS: Radio Access for Third Generation Mobile Communications (3rd Edition), Wiley, 2004.

2. Widmer, J., Handley, M. Extending equation-based congestion control to multicast Applications. In ACM SIGCOMM Computer Communication Review, 31, 4 (Aug 2001), 275-285.

3. Fu, C. P. and Liew S. C. TCP Veno: TCP enhancement for transmission over wireless access networks. IEEE Journal on Selected Areas in Communications, 54, 2 (Feb 2003), 216-228.

4. Liu, J., Li, B. and Zhang, Y.-Q. Adaptive video multicast over the Internet. IEEE Multimedia, 10, 1 (Jan-Mar 2003), 22-33.

5. Vickers, B., Albuquerque, C. and Suda, T. Source-adaptive multilayered multicast algorithms for real-time video distribution. [EEE/ACM Transactions on Networking, 8, 6 (Dec 2000), 720-732.

6. Donahoo, M. J. and Ainapure, S. R. Scalable multicast representative member selection. In Proceedings of the INFOCOM'01,(Anchorage, AK, USA, Apr 22-26, 2001), 259-268.

7. Saito, $T$. and Yamamoto, M. Wireless-caused representative selection fluctuation problem in wireless multicast congestion control. IECE Transactions on Communications, E88-B, 7 (Jul 2005), 2819-2825.

8. The Network Simulator - ns-2. Available online at: http://www.isi.edu/nsnam/ns.

9. Ericsson Telecommunicatie B.V. User Manual for EURANE, 2005. Available online at: http://www.ti-wmc.nl/eurane/eurane_user_guide_1 6.pdf.

10.Alexiou, A., Antonellis, D., Bouras, C., Papazois, A. An efficient multicast packet delivery scheme for UMTS. In Proceedings of the MSWiM'06, (Torremolinos, Malaga, Spain, Oct $2-6,2006), 147-150$. 